\title{
Initial central venous pressure could be a prognostic marker for hemodynamic improvement of polymyxin B direct hemoperfusion: a retrospective cohort study
}

Hiroyuki Yamada ${ }^{1,2 *}$, Tatsuo Tsukamoto ${ }^{1}$, Hiromichi Narumiya ${ }^{2,3}$, Kazumasa Oda $^{3}$, Satoshi Higaki ${ }^{3}$, Ryoji lizuka ${ }^{3}$, Motoko Yanagita ${ }^{1}$ and Masako Deguchi ${ }^{2}$

\begin{abstract}
Background: Direct hemoperfusion with polymyxin B-immobilized fiber column (PMX-DHP) could improve the hemodynamic status of septic shock patients. As PMX-DHP is an invasive and costly procedure, it is desirable to estimate the therapeutic effect before performing the therapy. However, it is still unclear when this therapy should be started and what type of sepsis it should be employed for. In this study, we retrospectively examined the clinical effect of patients treated with PMX-DHP by using central venous pressure (CVP).

Methods: Seventy patients who received PMX-DHP for septic shock during the study period were recruited and divided into a low CVP group $(n=33, C V P<12 \mathrm{mmHg})$ and a high CVP group $(n=37, \mathrm{CVP} \geqq 12 \mathrm{mmHg})$. The primary endpoint was vasopressor dependency index at 24 hours after starting PMX-DHP, and the secondary endpoint was the 28-day survival rate. Additionally, we performed a multivariate linear regression analysis on the difference in the vasopressor dependency index.

Results: The vasopressor dependency index significantly improved at $24 \mathrm{~h}$ in the low CVP group $\left(0.33\right.$ to $0.16 \mathrm{mmHg}^{-1}$; $p<0.01$ ) but not in the high CVP group (0.43 to $\left.0.34 \mathrm{mmHg}^{-1} ; p=0.41\right)$, and there was a significant difference between the two groups in the index at $24 \mathrm{~h}(p=0.02$ ). The 28-day survival rate was higher in the low CVP group (79 vs. $43 \% ; p<$ 0.01). Multivariate linear regression analysis showed that CVP $(p=0.04)$ was independently associated with the difference in the vasopressor dependency index.
\end{abstract}

Conclusions: Our study indicates that the clinical effect of PMX-DHP for septic shock patients with higher CVP ( $\geqq 12 \mathrm{mmHg}$ ) might be limited and that the initial CVP when performing PMX-DHP could function as an independent prognostic marker for the hemodynamic improvement.

Keywords: Polymyxin B, Hemoperfusion, Septic shock, Central venous pressure, PMX-DHP

\footnotetext{
* Correspondence: hyamada@kuhp.kyoto-u.ac.jp

'Department of Nephrology, Graduate School of Medicine, Kyoto University,

54 Shogoin-Kawahara-cho, Sakyo-ku, Kyoto 606-8507, Japan

${ }^{2}$ Department of Metabolism, Nephrology and Rheumatology, Japanese Red

Cross Kyoto Daini Hospital, 355-5 Haruobi, Kamigyo-ku, Kyoto 602-8026,

Japan

Full list of author information is available at the end of the article
} 


\section{Background}

Both the 2008 and 2012 Surviving Sepsis Campaign Guidelines (SSCG) recommend the rapid infusion of intravenous fluids until a central venous pressure (CVP) of $8-12 \mathrm{mmHg}$ is achieved during initial resuscitation $[1,2]$. However, many studies show that excess fluid accumulation is associated with adverse outcomes in critically ill patients [3-5]. In particular, positive fluid balance seems to be harmful for patients whose comorbid burden includes chronic heart failure and/or chronic kidney disease [6, 7]. In order to avoid excess volume expansion in those patients, it is important to carefully monitor intravascular volume by the following parameters: CVP, stroke volume variance, or extravascular lung water.

Direct hemoperfusion with a polymyxin B-immobilized fiber column (PMX-DHP), which can effectively adsorb bacterial endotoxin and lead to an earlier recovery from shock state, was first reported in 1994, and it has been used for the treatment of septic shock in many countries [8-11]. Although many clinical reports, including two randomized control trials, have shown the clinical effect of adapting PMX-DHP for septic shock patients, there is no clear consensus about the effect of the hemoperfusion [9-12]. As PMX-DHP is an invasive and costly procedure, it is desirable to accurately estimate the therapeutic effect before performing the therapy [11]. However, it is still unclear when this therapy should be started and what type of sepsis it should be employed for.

The utility of CVP as a marker of intravascular volume has been questioned for many years [13, 14]. However, we consider that CVP is one of the most widely used hemodynamic parameters because of the promptness of the measurement and the ability to perform it in any hospital facility. Actually, many clinical studies also demonstrated that high CVP was associated with positive fluid balance $[1,5,15]$.

In this study, in order to clarify the application of PMX-DHP for septic shock patients, we retrospectively examined the hemodynamic improvement and the mortality of patients treated with PMX-DHP by using CVP values. Moreover, we investigated whether the CVP values at the start of PMX-DHP could function as an independent prognostic factor for the hemodynamic improvement of the hemoperfusion.

\section{Methods}

\section{Patients}

We conducted a retrospective cohort study among all consecutive patients who received PMX-DHP for septic shock between May 2008 and April 2013 in the intensive care unit (ICU), high care unit (HCU), and cardiovascular care unit (CCU) at the Japanese Red Cross Kyoto Daini Hospital and the Kyoto University Hospital in
Japan. After initial resuscitation to achieve the early goal directed therapy (EGDT), PMX-DHP was applied along the Japanese health insurance system, as follows [1]: septic shock patients who require vasopressor support because of endotoxin or gram-negative bacteria. The following patients were excluded: (1) those who were under 18 years old, (2) those who were admitted to the ICU, HCU, or $\mathrm{CCU}$ for reasons other than sepsis, (3) those who were not given vasopressors when starting PMX-DHP, and (4) those in whose medical records CVP was not sufficiently recorded.

The Ethics Committee of Kyoto University Graduate School and Faculty of Medicine approved the protocol (E2153). This study was retrospective and used only a data bank while employing the highest privacy policy standards. Therefore, the requirement of informed consent was waived.

\section{Procedures}

Vascular access was placed at the femoral or the internal jugular vein. PMX-DHP with PMX-20R (Toray Industries, Tokyo, Japan) was performed for at least 120 min per session once or twice per patient per day for 2 days. The blood flow volume was $80-120 \mathrm{~mL} / \mathrm{min}$. The duration of hemoperfusion was decided by the attending physician. The therapy was terminated when the attending physician deemed it appropriate to conclude PMX-DHP for any reason. The anticoagulant used in PMX-DHP was nafamostat mesilate, low molecular weight heparin, or unfractionated heparin. All other cardiovascular management, including cardiac output management, setting of blood pressure goals, and fluid and inotropic therapy, were performed on the basis of SSCG recommendations by the attending physician.

\section{Definitions and classification}

In this study, we classified the patients into two groups: patients with CVP values greater than or equal to $(\geqq)$ $12 \mathrm{mmHg}$ when starting PMX-DHP were placed in the high CVP group, while the remaining patients whose CVP values were less than $(<) 12 \mathrm{mmHg}$ were placed in the low CVP group. CVP was measured using the standard method when starting PMX-DHP and expressed as $\mathrm{mmHg}$, as described previously [16, 17]. This classification is also based on the SSCG recommendations, which suggest that in mechanical ventilation patients, a higher target CVP of $12 \mathrm{mmHg}$ should be achieved [2].

\section{Data collection}

We employed three parameters in order to compare hemodynamic status among the patients in this study: mean arterial pressure (MAP), inotropic score, and vasopressor dependency index, as described in the 
preceding studies $[9,18]$. Namely, the inotropic score was calculated as follows:

$$
\begin{aligned}
& (\text { dopamine dose }[\mu \mathrm{g} / \mathrm{kg} / \mathrm{min}]) \\
& \quad \times 1+(\text { dobutamine }[\mu \mathrm{g} / \mathrm{kg} / \mathrm{min}]) \\
& \times 1+(\text { epinephrine dose }[\mu \mathrm{g} / \mathrm{kg} / \mathrm{min}]) \\
& \times 100+(\text { norepinephrine dose }[\mu \mathrm{g} / \mathrm{kg} / \mathrm{min}]) \\
& \times 100+(\text { phenylephrine dose }[\mu \mathrm{g} / \mathrm{kg} / \mathrm{min}]) \times 100
\end{aligned}
$$

And, vasopressor dependency index was calculated as the inotropic score/MAP. The parameters were calculated before the first PMX-DHP, immediately thereafter and $24 \mathrm{~h}$ after the first PMX-DHP.

Relevant clinical background, medical history, and clinical data of all patients were collected at appropriate times during the treatment for sepsis. Basic cardiopulmonary data and laboratory data obtained at the time of starting PMX-DHP were considered baseline values. These included age, sex, body mass index, systolic blood pressure, diastolic blood pressure, dopamine infusion rate, noradrenaline infusion rate, inotropic score, vasopressor dependency index, heart rate, central venous pressure, cardiac output, cardiac index, body temperature, arterial $\mathrm{pH}$, lactate, arterial oxygen tension $\left(\mathrm{PaO}_{2}\right)$ /fractional inspired oxygen $\left(\mathrm{FiO}_{2}\right)$ ratio ( $\mathrm{P} / \mathrm{F}$ ratio), positive end-expiratory pressure (PEEP), renal replacement therapy, surgery, hemoglobin, platelet count, C-reactive protein (CRP), total bilirubin, total protein, Acute Physiologic and Chronic Health Evaluation II (APACHE II) score, Sequential Organ Failure Assessment (SOFA) score, time from admission to care units until starting PMX-DHP, duration of PMXDHP, total fluid dosage from ICU admission until starting PMX-DHP, site of infection, and microorganism types. Cardiac output and cardiac index were measured by an arterial catheter attached to the Flotrac ${ }^{\mathrm{TM}}$ pulse counter device (Vigileo ${ }^{\mathrm{TM}}$, Edwards Lifesciences, Irvine, CA, USA) or a pulmonary artery catheter attached to Vigilance ${ }^{\mathrm{TM}}$ monitor (Vigileo ${ }^{\mathrm{TM}}$, Edwards Lifesciences, Irvine, CA, USA).

\section{Study outcomes}

The primary outcome was vasopressor dependency index at $24 \mathrm{~h}$ after starting PMX-DHP. The secondary outcome was the 28-day survival rate.

\section{Statistical analysis}

Statistical analysis was performed using JMP for Macintosh version 10.0.2 software (SAS Institute, Tokyo, Japan). Categorical variables are expressed as the number of patients (\%) and were analyzed by using the $\chi^{2}$ test or Fisher's exact test. Continuous variables are expressed as means and $95 \%$ confidence intervals (CIs). Comparison of continuous variables between the two groups was conducted with the $t$ test or the Mann-Whitney $U$ test, according to the distribution of the variables. Evaluation of significance between groups over time points was done by repeated measure ANOVA. As post hoc analysis, the three pair-wise comparisons of the hemodynamic status within a single group among different time points were made using Bonferroni adjustment. Therefore, $p$ values less than 0.016 were considered significant only in this comparison. In the other comparisons, statistical significance was defined as $p$ values $<0.05$. Kaplan-Meier curves were constructed for the comparison of the survival rate in the two groups and were tested for difference using the log-rank test.

To ensure the assumption that the CVP at the beginning of PMX-DHP could be an independently prognostic factor for the hemodynamic improvement of the hemoperfusion, we performed a multivariate linear regression analysis that focused on the difference in the vasopressor dependency index before PMX-DHP and 24 h after hemoperfusion. The relationships between the parameter and continuous variables were examined using Pearson's correlation coefficient, and categorical variables were examined using Spearman's R test. All variables with $p$ values $<0.20$ in the univariate analysis were included in the multivariate analysis. To ensure that the assumptions for regression analysis were not violated, an analysis of residuals was carried out. Moreover, we performed multivariate Cox regression analysis to assess the covariates that were associated with time to mortality.

\section{Results}

\section{Patient characteristics}

Although 112 patients received PMX-DHP for septic shock during the study period, 70 patients met the inclusion criteria, while 42 patients were excluded for a variety of reasons (age, 2; reasons for admission to ICU, 13; vasopressors not used, 9; no record of CVP, 18). Of these 70 patients, the initial CVP of 33 patients when receiving PMX-DHP was $<12 \mathrm{mmHg}$, and the initial CVP of the other 37 patients was $\geqq 12 \mathrm{mmHg}$, as shown in Fig. 1. The baseline characteristics of the study population are described in Table 1. Although the SOFA score, in particular SOFA liver and SOFA hematological, was significantly higher in the high CVP group than in the low CVP group, the APACHE II score was not significantly different between the two groups. Arterial $\mathrm{pH}$ was also significantly lower in the high CVP group. There were no significant differences in the other parameters except CVP. Although cardiac pump dysfunction could have a serious influence on CVP value, there was not a significant difference between the two groups in cardiac output and cardiac index before starting PMX-DHP. 


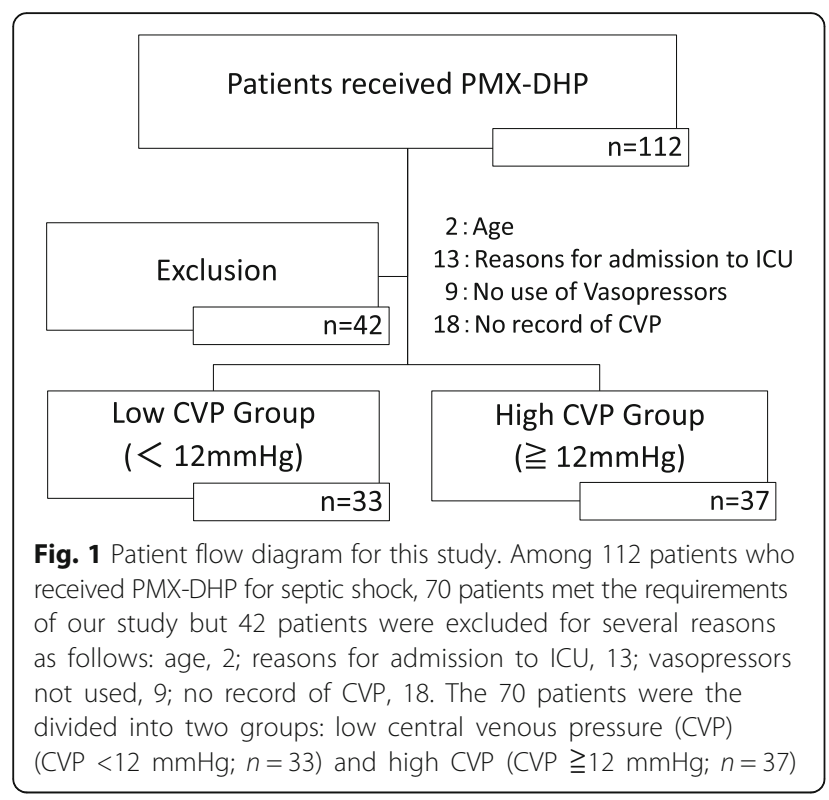

Table 2 shows the site of infection and microorganism types in both groups. There were also no significant differences between them.

\section{Primary outcome}

Repeated measures ANOVA for the vasopressor dependency index revealed that the index in the low CVP group improved more significantly than in the high CVP group (for each $p<0.01$ ) (Fig. 2). As for post hoc analysis, the vasopressor dependency index decreased significantly at 24. h $\left(0.16 \mathrm{mmHg}^{-1} ; 95 \% \mathrm{CI}, 0.05-0.28 ; p<0.01\right)$ but not after PMX-DHP $\left(0.24 \mathrm{mmHg}^{-1} ; 95 \% \mathrm{CI}, 0.15-0.34\right.$; $p=0.14)$ in the low CVP group whereas the decrease was observed neither after PMX-DHP $\left(0.39 \mathrm{mmHg}^{-1}\right.$; $95 \% \mathrm{CI}, 0.30-0.49 ; p=1.00)$ nor at $24 \mathrm{~h}\left(0.34 \mathrm{mmHg}^{-1}\right.$; $95 \% \mathrm{CI}, 0.25-0.44 ; p=0.41)$ in the high CVP group. Additionally, we could observe a significant difference in the index at $24 \mathrm{~h}$ between the two groups $(p<0.05)$ (Fig. 3). Thus, PMX-DHP appeared to be more effective for the hemodynamic status in the low CVP group than in the high CVP group.

\section{Secondary outcome}

The survival rates of both groups after 28 days were analyzed by the Kaplan-Meier method (Fig. 3). The survival rate was significantly higher in the low CVP group than in the high CVP group $(p<0.01)$, as determined by logrank test.

\section{Regression analysis}

Correlation analyses were performed to identify factors associated with the difference in the vasopressor dependency index before and $24 \mathrm{~h}$ after PMX-DHP (Additional file 1: Table S1). In the univariate regression analysis, age statistically significantly correlated with the difference $(p=0.03)$. Subsequent multivariate linear regression confirmed CVP $(p=0.04)$ and age $(p=$ 0.03 ) as independent prognosis factors regarding the hemodynamic improvement of PMX-DHP (Table 3). This also indicates that the higher the CVP at the start of hemoperfusion is, the less it improves the hemodynamic state.

\section{Discussion}

In this study, we observed the association between CVP and the hemodynamic improvement with PMX-DHP. Our results yielded two interesting findings. First, the hemodynamic status of patients with higher CVP did not improve significantly by PMX-DHP. In other words, our retrospective results did not support the guideline's recommendations, which suggested that septic shock patients with mechanical ventilation should achieve a higher target of CVP 12 to $15 \mathrm{mmHg}^{2}$. Second, the initial CVP when performing PMX-DHP could function as an independent prognostic factor for the hemodynamic improvement of the therapy. To the best of our knowledge, this is the first study that investigated this particular association and prognosis.

Although CVP is one of the most popular hemodynamic parameters, we cannot deny that CVP values may not reflect intravascular volume accurately. In fact, recent reviews reported that the CVP value is mainly determined by two factors: cardiac pump function and venous return function $[17,19,20]$. In terms of cardiac function, a high CVP indicates a decrease in contractility, diastolic dysfunction, valvular disease, and cardiomyopathy in these patients, although in our study, there was not a significant difference in cardiac output and cardiac index $[19,20]$. On the other hand, venous return is determined by the gradient between CVP and the mean circulatory filling pressure (MCFP), as shown in the formula below:

$$
\text { venous return }=(\mathrm{MCFP}-\mathrm{CVP}) / \text { venous resistance }
$$

[19] MCFP is the pressure in the vasculature when the heart is stopped (zero flow) and the pressures in all segments of the circulatory system have equalized [21, 22]. Thus, an increase in CVP values leads to the decrease in venous return [19-22]. Because PMX-DHP does not directly affect these pressures and cardiac function, it is difficult for the hemoperfusion to improve the hemodynamic status for septic shock patients with high CVP.

Actually, in this study, we observed that the patients in the high CVP group suffered from hemodynamic impairment due to high CVP. The proportion of patients who received renal replacement therapy was non-significantly larger in the high CVP group, which suggests that many 
Table 1 Baseline characteristics of the patients

\begin{tabular}{|c|c|c|c|}
\hline \multirow{3}{*}{ Age, year } & \multirow{2}{*}{$\begin{array}{l}\text { Low CVP group } n=33 \\
\text { Median }(95 \% \mathrm{Cl})\end{array}$} & \multirow{2}{*}{$\begin{array}{l}\text { High CVP group } n=37 \\
\text { Median }(95 \% \mathrm{Cl})\end{array}$} & \multirow[t]{2}{*}{$p$ value } \\
\hline & & & \\
\hline & $72(68-76)$ & $67(64-71)$ & 0.07 \\
\hline & $20(61)$ & $26(70)$ & 0.46 \\
\hline Body mass index, $\mathrm{kg} / \mathrm{m}^{2}$ & $22(20-23)$ & $23(21-24)$ & 0.37 \\
\hline Systolic blood pressure, $\mathrm{mmHg}$ & $99(90-106)$ & $92(84-99)$ & 0.22 \\
\hline Diastolic blood pressure, $\mathrm{mmHg}$ & $50(47-53)$ & $48(44-51)$ & 0.30 \\
\hline Mean blood pressure, $\mathrm{mmHg}$ & $66(62-70)$ & $62(59-66)$ & 0.17 \\
\hline Dopamine infusion rate, $\mu \mathrm{g} / \mathrm{kg} / \mathrm{min}$ & $5.0(3.5-6.6)$ & $5.8(4.4-7.3)$ & 0.45 \\
\hline Noradrenaline infusion rate, $\mu \mathrm{g} / \mathrm{kg} / \mathrm{min}$ & $0.12(0.09-0.16)$ & $0.16(0.12-0.20)$ & 0.20 \\
\hline Inotropic score & $20(15-25)$ & $25(21-30)$ & 0.14 \\
\hline Vasopressor dependency index, $\mathrm{mmHg}^{-1}$ & $0.33(0.23-0.45)$ & $0.43(0.34-0.53)$ & 0.15 \\
\hline Heart rate, bpm & $109(103-116)$ & $115(109-121)$ & 0.18 \\
\hline $\mathrm{CVP}, \mathrm{mmHg}$ & $8(7-9)$ & $15(14-16)$ & $<0.01$ \\
\hline Cardiac output, L/min & $4.6(3.3-5.8)$ & $5.7(4.6-5.7)$ & 0.18 \\
\hline Cardiac index, $\mathrm{L} / \mathrm{min} / \mathrm{m}^{2}$ & $2.8(2.3-3.4)$ & $3.3(2.8-3.7)$ & 0.23 \\
\hline Body temperture, ${ }^{\circ} \mathrm{C}$ & $36.8(36.4-37.2)$ & $36.8(36.4-37.2)$ & 0.92 \\
\hline Arterial pH & $7.36(7.32-7.41)$ & $7.27(7.23-7.31)$ & $<0.01$ \\
\hline Lactate, $\mathrm{mmol} / \mathrm{L}$ & $3.9(2.6-5.3)$ & $4.0(2.9-5.2)$ & 0.94 \\
\hline $\mathrm{P} / \mathrm{F}$ ratio & $220(181-258)$ & $168(131-206)$ & 0.06 \\
\hline PEEP, $\mathrm{cmH}_{2} \mathrm{O}$ & $8(6-10)$ & $9(7-11)$ & 0.32 \\
\hline Renal replacement therapy, $n(\%)$ & $13(39)$ & $23(62)$ & 0.06 \\
\hline Surgery, $n(\%)$ & $21(64)$ & $17(46)$ & 0.16 \\
\hline Hemoglobin, g/dL & $10.4(9.7-11.1)$ & $10.3(9.6-11.0)$ & 0.78 \\
\hline Platelet count, $\times 10^{9} / \mathrm{L}$ & $111(87-134)$ & $81(59-104)$ & 0.08 \\
\hline $\mathrm{CRP}, \mathrm{mg} / \mathrm{dL}$ & $17.7(11.4-24.0)$ & $17.6(11.6-23.5)$ & 0.97 \\
\hline Total Bilirubin, mg/dL & $1.7(0.2-3.1)$ & $3.6(2.2-5.0)$ & 0.06 \\
\hline Total Protein, mg/dL & $4.9(4.5-5.3)$ & $4.6(4.2-4.9)$ & 0.17 \\
\hline APACHE II score & $26(25-28)$ & $28(26-30)$ & 0.23 \\
\hline SOFA score & $12(11-13)$ & $14(13-15)$ & 0.01 \\
\hline SOFA cardiovascular & $3.5(3.3-3.7)$ & $3.7(3.5-3.9)$ & 0.29 \\
\hline SOFA renal & $1.9(1.4-2.4)$ & $2.3(1.8-2.8)$ & 0.22 \\
\hline SOFA hematological & $1.6(1.2-2.0)$ & $2.1(1.8-2.5)$ & 0.05 \\
\hline SOFA respiratory & $2.4(1.9-2.8)$ & $2.8(2.4-3.2)$ & 0.15 \\
\hline SOFA liver & $0.8(0.4-1.2)$ & $1.4(1.0-1.7)$ & 0.04 \\
\hline SOFA central nerve system & $2.0(1.6-2.5)$ & $2.0(1.5-2.4)$ & 0.83 \\
\hline Time from ICU admission until starting PMX-DHP, min & $441(98-802)$ & $829(498-1159)$ & 0.11 \\
\hline PMX-DHP duration, min & $354(249-458)$ & $366(263-469)$ & 0.97 \\
\hline Total fluid dosage from ICU admission until starting PMX-DHP, ml & $2970(1200-4760)$ & $3212(1223-5203)$ & 0.86 \\
\hline
\end{tabular}

APACHE Acute Physiologic and Chronic Health Evaluation, CRP c-reactive protein, CVP central venous pressure, ICU intensive care unit, $P E E P$ positive end-expiratory pressure, $P / F$ ratio arterial oxygen tension/fractional inspired oxygen ratio $P M X$-DHP direct hemoperfusion with polymyxin B-immobilized fiber column, SOFA Sequential Organ Failure Assessment

of the attending physicians might think the intravascular volume in the high CVP group patients is too large. Additionally, the P/F ratio and PEEP were also nonsignificantly lower in the high CVP group, which indicated that some of the patients had a high intrathoracic pressure. Hence, we consider that high CVP group patients did not have adequate venous return because excess fluid therapy or high intrathoracic pressure reduces the 
Table 2 Isolated microorganisms by treatment group

\begin{tabular}{|c|c|c|}
\hline & $\begin{array}{l}\text { Low CVP group } \\
n=33\end{array}$ & $\begin{array}{l}\text { High CVP group } \\
n=37\end{array}$ \\
\hline \multicolumn{3}{|l|}{ Site of infection } \\
\hline Abdomen & 13 & 15 \\
\hline Lung & 6 & 13 \\
\hline Urinary tract & 7 & 2 \\
\hline Skin & 2 & 1 \\
\hline Blood stream & 0 & 1 \\
\hline Others & 5 & 5 \\
\hline \multicolumn{3}{|l|}{ Microorganism type } \\
\hline Escherichia coli & 7 & 5 \\
\hline Staphylococcus species & 2 & 5 \\
\hline Streptococcus species & 1 & 4 \\
\hline Enterococcus species & 1 & 2 \\
\hline Pseudomonas species & 1 & 2 \\
\hline Bacteroides species & 1 & 1 \\
\hline Klebsiella species & 2 & 0 \\
\hline Serratia species & 1 & 1 \\
\hline Acinetobacter species & 1 & 0 \\
\hline Citrobacter species & 0 & 1 \\
\hline Clostridium species & 1 & 0 \\
\hline Morallexa species & 0 & 1 \\
\hline Stenotrophomonas species & 0 & 1 \\
\hline
\end{tabular}

gradient between MCFP and CVP. On the other hand, the significant improvement in the low CVP group could be because they genuinely received the clinical effect of PMX-DHP. Generally, PMX-DHP can reduce plasma cytokine levels by absorbing endotoxin, immune cells, and anandamide [23-25]. These physiological and pathological responses could be equivalent in both groups in our study. However, the harmful effect of high CVP at the start of PMX-DHP differentiated the clinical effect of both groups. In other words, fluid toxicity or increase in intrathoracic pressure might be deleterious beyond the beneficial effect of PMX-DHP in patients with high CVP in our study.

Previous studies have reported that early initiation of PMX-DHP reduced the catecholamine requirement and that early improvement in inotropic score and vasopressor dependency index after PMX-DHP might be a prognostic factor $[18,23,26]$. In our study, the patients in the low CVP group who received PMXDHP earlier also tended to show a decrease in their vasopressor dependency index. Meanwhile, in terms of the time between ICU admission and starting PMX-DHP, there was neither a statistical difference between the two groups nor a significant association with hemodynamic improvement in our study. However, because the sample size of our study was not large

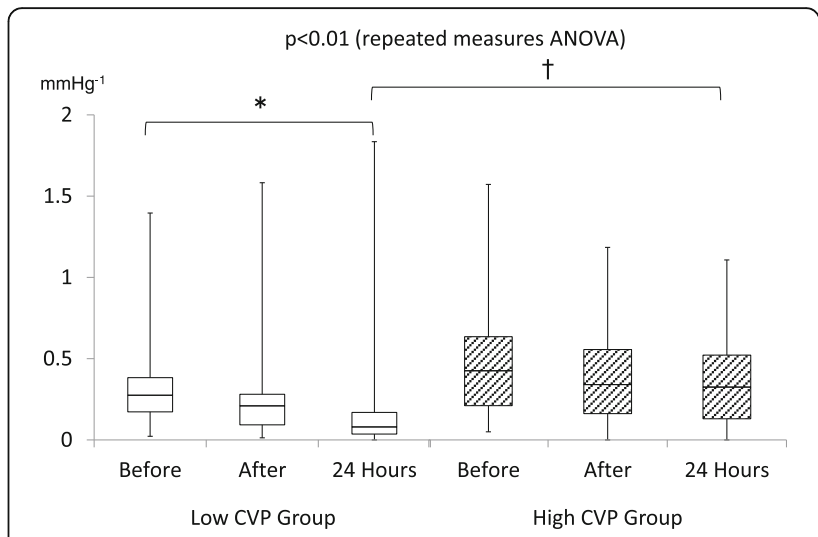

Fig. 2 Changes in vasopressor dependency index. Each box plot indicates medians, 25th and 75th percentiles, and whisker caps indicate 5th and 95th percentiles. White box plots indicate the low CVP group, and the diagonal-lined box indicates the high CVP group. An asterisk indicates a significant difference with $p<0.01$, and a dagger indicates a significant difference with $p<0.05$. There was a significant difference between the two groups in the repeated measure ANOVA. The vasopressor dependency index also significantly improved at $24 \mathrm{~h}$ in the low CVP group (0.28 to $\left.0.16 \mathrm{mmHg}^{-1} ; p<0.01\right)$ but not in the high CVP group (0.43 to $0.34 \mathrm{mmHg}^{-1} ; p=0.10$ ). Additionally, there was a significant difference in the index at $24 \mathrm{~h}$ between the two groups $(p<0.05)$

enough to demonstrate the association, our results should be viewed with this limitation in mind. In addition, although we performed PMX-DHP for around $6 \mathrm{~h}$ in both groups, a recent study has indicated that a longer duration of PMX-DHP therapy can be expected to improve the hemodynamics and pulmonary oxygenation capacity of patients with severe sepsis/septic shock [27]. Thus, longer operation of PMX-DHP might contribute to improve the outcome of patients with low CVP.

Other limitations of our study need to be acknowledged. First, we could not show the data on intrinsic PEEP, so-

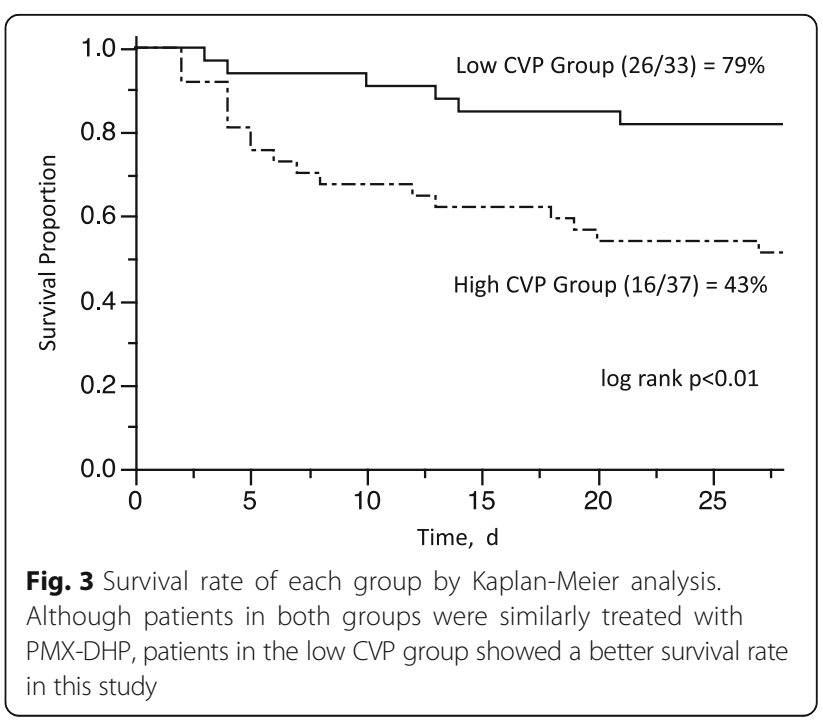


Table 3 Results of multivariate regression analysis for the difference in the vasopressor dependency index between before and $24 \mathrm{~h}$ after PMX-DHP

\begin{tabular}{lcccc}
\hline Selected variables & Regression coefficient $(\beta)$ & $95 \% \mathrm{Cl}$ & Partial correlations & $p$ value \\
\hline Age & -0.2972 & $-0.0168--0.0009$ & -0.0089 & 0.03 \\
CVP & -0.3155 & $-0.0348--0.0007$ & -0.0178 & 0.180 \\
SOFA & 0.1530 & $-0.0166-0.0497$ & 0.0165 & 0.32 \\
Arterial pH & -0.2871 & $-1.2656--0.0171$ & -0.6242 & 0.06 \\
\hline
\end{tabular}

CVP central venous pressure, PMX-DHP direct hemoperfusion with polymyxin B-immobilized fiber column, SOFA Sequential Organ Failure Assessment

called auto-PEEP, which might have a direct influence on the CVP values in the high CVP group. However, we consider that it may not fundamentally change our conclusion. Auto-PEEP-induced hypotension is not a result of hyper-inflammatory response to sepsis, but it is rather a patient-ventilator interaction. Thus, it is obvious that PMX-DHP is less effective for the high CVP patients with auto-PEEP-induced hypotension. Additionally, this clinical study is not for acute exacerbation of chronic obstructive pulmonary disease, bronchial asthma, or acute respiratory distress syndrome, and it only evaluated patients with septic shock. Thus, we consider that there were not a large proportion of the study patients with auto-PEEP in our study. Second, the previous studies reported that high CVP was associated with a poor prognosis [28, 29]. Indeed, high CVP might have a negative influence on the cardiac function during the treatment in patients with high CVP group, although cardiac output and index were not significantly different in both groups at the beginning of the hemoperfusion [29]. Therefore, regardless of the effect of PMX-DHP, there may be a possibility of observing the clinical course of patients with a poor prognosis. Third, this study was not a randomized controlled trial, and we cannot rule out the possibility of selection bias, especially referral bias and Neyman bias. Fourth, perhaps we could not extract the patients whose conditions changed rapidly or whose case was extremely severe because our study patients had enough time to receive the hemoperfusion. Fifth, vasopressors were regulated by local physicians, depending on the patient's condition. Therefore, the protocol for titrating the vasopressors was different among the attending physicians. Further study is required to clarify these unsolved issues.

\section{Conclusions}

Our study indicated that the effect of PMX-DHP for septic shock patients with higher CVP $(\geqq 12 \mathrm{mmHg})$ may be limited and that the initial CVP in performing PMX-DHP could be an independent prognostic marker for hemodynamic improvement. Further study is required to clarify the mechanisms of PMX-DHP that affect sepsis treatment.

\section{Additional file}

Additional file 1: Table S1. Results of univariate regression analysis for the difference in the vasopressor dependency index between before and $24 \mathrm{~h}$ after PMX-DHP. (XLSX $12 \mathrm{~kb})$

\section{Abbreviations}

APACHE: Acute Physiologic and Chronic Health Evaluation;

CCU: Cardiovascular care unit; CRP: C-reactive protein; CVP: Central venous pressure; EGDT: Early goal directed therapy; ER: Emergency room; HCU: High care unit; ICU: Intensive care unit; MAP: Mean arterial pressure; MCFP: Mean circulatory filling pressure; P/F ratio: Arterial oxygen tension/fractional inspired oxygen ratio; PEEP: Positive end-expiratory pressure; PMXDHP: Direct hemoperfusion with polymyxin B-immobilized fiber column; SOFA: Sequential Organ Failure Assessment; SSCG: Surviving Sepsis Campaign Guidelines

\section{Acknowledgements}

We thank Juan Alejandro Oliva Trejo (Medical Innovation Center, TMK project, Graduate School of Medicine, Kyoto University) for serving as an advisor and the medical staff of the intensive care unit, high care unit, and cardiac care unit of both the Japanese Red Cross Kyoto Daini Hospital and Kyoto University Hospital.

\section{Funding}

None of the authors received any funding for this study.

\section{Availability of supporting data}

The datasets supporting the conclusions of this article are included within the article and in Additional file 1.

\section{Authors' contributions}

HY designed the study protocols, acquired the data, performed the statistical analysis, and completed the manuscript for publication. $\Pi$ revised the manuscript and approved it for publication. $\mathrm{HN}$ and $\mathrm{KO}$ helped with the data analysis. $\mathrm{SH}, \mathrm{RI}, \mathrm{MY}$, and MD supervised the interpretation of the results and writing of the reports. All authors have read and approved the final version of the manuscript.

\section{Competing interests}

The authors declare that they have no competing interests.

\section{Consent for publication}

Not applicable.

\section{Ethics approval and consent to participate}

This study was conducted in accordance with the principles of the Declaration of Helsinki because our research analyzed human data. Our research protocol was approved by Ethics Committee of Kyoto University Graduate School and Faculty of Medicine (E2153). This study was retrospective and used only a data bank while employing the highest privacy policy standards. Therefore, the requirement of informed consent was waived. 


\section{Previous presentations}

This study was presented in part at the 10th International Society for Apheresis Congress May 15, 2015, Cancun, Mexico, and at the 26th Annual Meeting of the Japan Society for Blood Purification in Critical Care October 9, 2015, Tokyo, Japan.

\section{Author details}

'Department of Nephrology, Graduate School of Medicine, Kyoto University, 54 Shogoin-Kawahara-cho, Sakyo-ku, Kyoto 606-8507, Japan. ${ }^{2}$ Department of Metabolism, Nephrology and Rheumatology, Japanese Red Cross Kyoto Dain Hospital, 355-5 Haruobi, Kamigyo-ku, Kyoto 602-8026, Japan. ${ }^{3}$ Department of Emergency, Japanese Red Cross Kyoto Daini Hospital, 355-5 Haruobi, Kamigyo-ku, Kyoto 602-8026, Japan.

\section{Received: 12 April 2016 Accepted: 15 September 2016} Published online: 10 October 2016

\section{References}

1. Rivers E, Nguyen B, Havstad S, et al. Early goal-directed therapy in the treatment of severe sepsis and septic shock. N Engl J Med. 2001;345(19):1368-77.

2. Dellinger RP, Levy MM, Rhodes $A$, et al. Surviving sepsis campaign. Crit Care Med. 2013;41(2):580-637.

3. Wiedemann HP, Wheeler AP, Bernard GR, et al. Comparison of two fluid-management strategies in acute lung injury. N Engl J Med. 2006;354(24):2564-75.

4. Bouchard J, Soroko SB, Chertow GM, et al. Fluid accumulation, survival and recovery of kidney function in critically ill patients with acute kidney injury. Kidney Int. 2009;76(4):422-7.

5. Boyd JH, Forbes J, Nakada T-a, Walley KR, Russell JA. Fluid resuscitation in septic shock: a positive fluid balance and elevated central venous pressure are associated with increased mortality*. Crit Care Med. 2011;39(2):259-65.

6. Yancy CW, Jessup M, Bozkurt B, et al. 2013 ACCF/AHA guideline for the management of heart failure: a report of the American College of Cardiology Foundation/American Heart Association Task Force on Practice Guidelines. J Am Coll Cardiol. 2013:62(16):e147-239.

7. Tsai YC, Tsai JC, Chen SC, et al. Association of fluid overload with kidney disease progression in advanced CKD: a prospective cohort study. Am J Kidney Dis. 2014;63(1):68-75.

8. Shoji H. Extracorporeal endotoxin removal for the treatment of sepsis: endotoxin adsorption cartridge (toraymyxin). Ther Apher Dial. 2003;7(1):108-14.

9. Cruz DN, Antonelli M, Fumagalli $R$, et al. Early use of polymyxin B hemoperfusion in abdominal septic shock: the EUPHAS randomized controlled trial. JAMA. 2009;301(23):2445-52

10. Berto P, Ronco C, Cruz D, Melotti RM, Antonelli M. Cost-effectiveness analysis of polymyxin-B immobilized fiber column and conventional medical therapy in the management of abdominal septic shock in Italy. Blood Purif. 2011;32(4):331-40.

11. Cruz DN, Perazella MA, Bellomo R, et al. Effectiveness of polymyxin Bimmobilized fiber column in sepsis: a systematic review. Crit Care. 2007:11(2):R47.

12. Payen DM, Guilhot J, Launey $Y$, et al. Early use of polymyxin B hemoperfusion in patients with septic shock due to peritonitis: a multicenter randomized control trial. Intensive Care Med. 2015;41(6):975-84.

13. Hoffman MJ, Greenfield LJ, Sugerman HJ, Tatum JL. Unsuspected right ventricular dysfunction in shock and sepsis. Ann Surg. 1983;198(3):307-19.

14. Marik PE, Cavallazzi R. Does the central venous pressure predict fluid responsiveness? An updated meta-analysis and a plea for some common sense*. Crit Care Med. 2013;41(7):1774-81.

15. Van Biesen W, Yegenaga I, Vanholder R, et al. Relationship between fluid status and its management on acute renal failure (ARF) in intensive care unit (ICU) patients with sepsis: a prospective analysis. J Nephrol. 2005;18(1):54-60.

16. Magder S. Central venous pressure: a useful but not so simple measurement. Crit Care Med. 2006;34(8):2224-7.

17. Magder S, Bafaqeeh F. The clinical role of central venous pressure measurements. J Intensive Care Med. 2007;22(1):44-51.

18. Kobayashi A, Iwasaki Y, Kimura Y, Kawagoe Y, Ujike Y. Early recovery in hemodynamics after direct hemoperfusion with polymyxin B-immobilized fibers may predict mortality rate in patients with septic shock. J Anesth. 2010;24(5):709-15.
19. Gelman S. Venous function and central venous pressure: a physiologic story. Anesthesiology. 2008;108(4):735-48.

20. Magder S. Bench-to-bedside review: an approach to hemodynamic monitoring-Guyton at the bedside. Crit Care. 2012;16(5):236.

21. Marik PE. latrogenic salt water drowning and the hazards of a high central venous pressure. Ann Intensive Care. 2014;4:21.

22. Henderson WR, Griesdale DE, Walley KR, Sheel AW. Clinical review: Guyton-the role of mean circulatory filling pressure and right atrial pressure in controlling cardiac output. Crit Care. 2010;14(6):1.

23. Ikeda T, Ikeda K, Nagura M, et al. Clinical evaluation of PMX-DHP for hypercytokinemia caused by septic multiple organ failure. Ther Apher Dial. 2004;8(4):293-8.

24. Nishibori M, Takahashi HK, Katayama H, et al. Specific removal of monocytes from peripheral blood of septic patients by polymyxin B-immobilized filter column. Acta Med Okayama. 2009:63(1):65-9.

25. Kohro S, Imaizumi H, Yamakage $M$, et al. Anandamide absorption by direct hemoperfusion with polymixin B-immobilized fiber improves the prognosis and organ failure assessment score in patients with sepsis. J Anesth. 2006:20(1):11-6.

26. Takeyama N, Noguchi H, Hirakawa A, et al. Time to initiation of treatment with polymyxin B cartridge hemoperfusion in septic shock patients. Blood Purif. 2012;33(4):252-6.

27. Yamashita C, Hara Y, Kuriyama N, Nakamura T, Nishida O. Clinical effects of a longer duration of polymyxin B-immobilized fiber column direct hemoperfusion therapy for severe sepsis and septic shock. Ther Apher Dial. 2015;19(4):316-23.

28. Wang XT, Yao B, Liu DW, Zhang HM. Central venous pressure dropped early is associated with organ function and prognosis in septic shock patients: a retrospective observational study. Shock. 2015;44(5):426-30.

29. Damman K, van Deursen VM, Navis G, Voors AA, van Veldhuisen DJ, Hillege $\mathrm{HL}$. Increased central venous pressure is associated with impaired renal function and mortality in a broad spectrum of patients with cardiovascular disease. J Am Coll Cardiol. 2009:53(7):582-8.

\section{Submit your next manuscript to BioMed Central and we will help you at every step:}

- We accept pre-submission inquiries

- Our selector tool helps you to find the most relevant journal

- We provide round the clock customer support

- Convenient online submission

- Thorough peer review

- Inclusion in PubMed and all major indexing services

- Maximum visibility for your research

Submit your manuscript at www.biomedcentral.com/submit
) Biomed Central 